\title{
Abelian duality, walls and boundary conditions in diverse dimensions
}

\author{
Anton Kapustin ${ }^{a}$ and Mikhail Tikhonov ${ }^{b}$ \\ ${ }^{a}$ California Institute of Technology, \\ Pasadena, CA 91125, United States \\ ${ }^{b}$ Département de physique, École Normale Supérieure, \\ 75231 Paris Cedex 05, France \\ E-mail: kapustin@theory.caltech.edu, tikhonov@princeton.edu
}

\begin{abstract}
We systematically apply the formalism of duality walls to study the action of duality transformations on boundary conditions and local and nonlocal operators in two, three, and four-dimensional free field theories. In particular, we construct a large class of D-branes for two-dimensional sigma-models with toroidal targets and determine the action of the T-duality group on it. It is manifest in this formalism that T-duality transformations on D-branes are given by a differential-geometric version of the Fourier-Mukai transform.
\end{abstract}

Keywords: Duality in Gauge Field Theories, Chern-Simons Theories, D-branes

ARXIV EPRINT: 0904.0840 


\section{Contents}

1 Introduction $\quad 1$

2 Abelian duality in 4d 2

2.1 Boundary conditions for a U(1) gauge theory 2

$2.2 \mathrm{SL}(2, \mathbb{Z})$ action on boundary conditions 5

2.3 The action of duality on operators 8

2.3.1 Wilson and 't Hooft loops 9

$\begin{array}{lll}2.3 .2 & \text { Chern-Simons operator } & 12\end{array}$

3 Abelian duality in 2d 13

$\begin{array}{ll}3.1 \text { Toroidal sigma-model } & 13\end{array}$

$\begin{array}{lll}3.2 & \text { Boundary conditions for the sigma-model } & 15\end{array}$

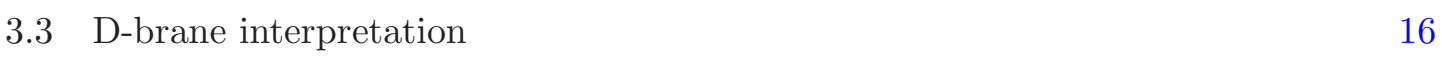

$\begin{array}{lll}3.4 & \text { Duality action on boundary conditions } & 18\end{array}$

4 Abelian duality in 3d $\quad 20$

4.1 The duality wall in 3d 20

$\begin{array}{lll}4.2 & \text { Duality action on operators } & 23\end{array}$

$\begin{array}{ll}4.3 & \text { Duality action on boundary conditions }\end{array}$

\section{Introduction}

It has been realized recently (see e.g. $[2-4,10,12]$ ) that the most economic description of duality transformations in field theory is by means of codimension-1 defects (walls). Any equivalence between quantum field theories $\mathrm{A}$ and $\mathrm{B}$ gives rise to a domain wall which separates a domain described by the field theory $\mathrm{A}$ and a domain described by the field theory B. We will refer to these domains as phase A and phase B. While there may be many different domain walls between these phases, the wall corresponding to a duality transformation has a very special property: it is topological, in the sense that correlation functions are unchanged if one deforms the location of the wall without crossing the insertion points of any operators. Given such a wall, one can determine how the duality acts on any local operator $\mathcal{O}$ in the theory A: one simply considers a "composite" made of $\mathcal{O}$ and the wall wrapping a small sphere centered at the insertion point of $\mathcal{O}$, so that the interior of the sphere is in phase A, while the exterior is in phase B. In the limit when the radius of the sphere goes to zero, this composite defines a local operator in the theory $\mathrm{B}$. Similarly, one can determine how nonlocal operators and boundary conditions transform under duality transformations. 
In this paper we systematically apply these ideas to abelian dualities in two, three, and four dimensions. In $4 \mathrm{~d}$ we study duality transformations for the free $\mathrm{U}(1)$ gauge theory. There is a substantial overlap here with a recent work of Gaiotto and Witten [4]. For example, Gaiotto and Witten explain how to find the electric-magnetic dual of an arbitrary boundary condition in $4 \mathrm{~d} \mathrm{U}(1)$ gauge theory. Our contribution is to provide some examples of using the wall formalism to determine the mapping of nonlocal operators under the $\operatorname{SL}(2, \mathbb{Z})$ duality group. For example, we explain how to find the dual of the Chern-Simons operator in the $4 \mathrm{~d} \mathrm{U}(1)$ gauge theory, something which is difficult to do using the traditional methods.

In the two-dimensional case, we show how the formalism of duality walls enables us to define the action of T-duality on a large class of branes on tori in a uniform way. For example, we show by a simple manipulation of the path-integral that the T-dual of a vector bundle on $T^{2}$ of rank $r$ and first Chern class $k$ is a vector bundle on the dual torus of rank $k$ and first Chern class $-r$. Boundary degrees of freedom describing the Chan-Paton labels appear naturally in this derivation. This is an improvement over the traditional argument which uses step-wise T-duality (dualizing circles one by one). More generally, we show that for our class of D-branes T-duality acts as a differential-geometric version of the FourierMukai transform. Domain walls corresponding to T-dualities have been recently discussed in [12]. The connection between T-duality and Fourier-Mukai transform has also been noted in the literature, but usually in the context of supersymmetric sigma-models and supersymmetric branes. We do not make use of supersymmetry in this paper.

In the three-dimensional case the abelian duality relates a $\mathrm{U}(1)$ gauge theory with a Maxwell action and a massless scalar field. We describe the wall corresponding to this duality and use it to deduce how the duality acts on some boundary conditions and operators. Somewhat unexpectedly, to define the duality wall precisely one needs to make use of the notion of a gerbe connection (also known as a B-field). We also discuss how the introduction of the Chern-Simons term in the gauge theory destroys the duality.

M. T. would like to thank California Institute of Technology for hospitality during the initial stages of this work. A. K. was supported in part by the DOE grant DE-FG03-92ER40701.

\section{Abelian duality in $4 d$}

\subsection{Boundary conditions for a U(1) gauge theory}

We will study a $\mathrm{U}(1)$ gauge theory on a Riemannian 4-manifold $M$ with an action

$$
S=\frac{1}{2 e^{2}} \int_{M} F \wedge * F+\frac{i \theta}{8 \pi^{2}} \int_{M} F \wedge F
$$

Here $F=d A$ is the curvature of the $\mathrm{U}(1)$ gauge field $A$. The simplest boundary condition for the gauge field is to require the restriction of $A$ to $\partial M$ to be trivial (i. e. gaugeequivalent to zero). More generally, we can require $\left.A\right|_{\partial M}$ to be a flat, but not necessarily trivial (fixed) connection. The curvature then satisfies

$$
\left.F\right|_{\partial M}=0 .
$$


If the boundary contains a time-like direction, then this condition means, in three-dimensional terms, that the electric field is normal to the boundary, while the magnetic field is tangent to it. Thus it corresponds to a perfect electric conductor (PEC).

Another simple condition is the free boundary condition which allows $\left.A\right|_{\partial M}$ to be unconstrained. The vanishing of the boundary part of the variation of the action then gives the following boundary condition on the curvature:

$$
\left.\frac{1}{e^{2}} * F\right|_{\partial M}+\left.\frac{i \theta}{4 \pi^{2}} F\right|_{\partial M}=0 .
$$

For $\theta=0$ it means that the electric field is tangent to the (space-like) boundary, while the magnetic field is normal to it. This corresponds to a magnetic with an infinitely large magnetic permeability. Since the perfect electric conductor is formally equivalent to a dielectric with an infinite electric permeability, it is natural to regard the boundary condition (2.2) as corresponding to a "perfect magnetic conductor" (PMC).

This terminology is also natural because the PEC and PMC boundary conditions are related by electric-magnetic duality. This is rather obvious for $\theta=0$ since electric-magnetic duality for $\theta=0$ exchanges electric and magnetic fields. It is also true for arbitrary $\theta$ as we will see below.

As explained above, on the quantum level the PEC condition has moduli which are described by a flat $\mathrm{U}(1)$ connection on $\partial M$. Neglecting the torsion phenomena, the moduli space of such connections is a torus

$$
H^{1}(\partial M, \mathbb{R}) / 2 \pi H^{1}(\partial M, \mathbb{Z})
$$

There are also moduli in the PMC case. We can add to the action a boundary term

$$
\frac{i}{2 \pi} \int_{\partial M} F \wedge \lambda
$$

where $\lambda$ is a closed 1 -form on $\partial M .^{1}$ Since the periods of $F$ are integers modulo $2 \pi$, the action is invariant under

$$
\lambda \mapsto \lambda+2 \pi \alpha,
$$

where $\alpha$ is a closed 1-form on $\partial M$ with integral periods. The set of closed 1-forms modulo such transformations is again the torus

$$
H^{1}(\partial M, \mathbb{R}) / 2 \pi H^{1}(\partial M, \mathbb{Z})
$$

We will see below that PEC and PMC moduli are exchanged by electric-magnetic duality.

To realize the full duality group $\operatorname{SL}(2, \mathbb{Z})$ one has to consider more general boundary conditions. In fact, to realize $\mathrm{SL}(2, \mathbb{Z})$ quantum-mechanically one has to allow boundary degrees of freedom. These degrees of freedom are topological, in the sense that in the limit $e \rightarrow 0$ they are described by a $3 \mathrm{~d}$ topological gauge theory on $\partial M$.

\footnotetext{
${ }^{1}$ The form $\lambda$ is taken to be closed so that the extra term does not contribute to the boundary variation of the action and therefore does not affect the boundary condition (2.2).
} 
A natural $3 \mathrm{~d}$ topological field theory which can be coupled to a $\mathrm{U}(1)$ gauge theory is abelian Chern-Simons theory [14]. Its action is

$$
\sum_{j l} \frac{i \mathrm{k}_{j l}}{4 \pi} \int_{\partial M} a^{j} d a^{l} .
$$

where the indices $j, l$ run from 1 to $n$ and $a^{j}$ is a connection 1-form on a $\mathrm{U}(1)$ bundle over $\partial M$. The matrix $\mathrm{k}_{j l}$ is symmetric and integral; this ensures the invariance of the path-integral under large gauge transformations. This $3 \mathrm{~d}$ theory has conserved currents

$$
J^{l}=* \frac{1}{2 \pi} d a^{l}, \quad l=1, \ldots, n .
$$

The corresponding charges are quantized:

$$
\int_{\Sigma} * J^{l} \in \mathbb{Z}
$$

where $\Sigma$ is a 2-cycle in $\partial M$. These charges are simply fluxes of the gauge fields $a^{j}$.

To couple such a topological gauge theory to a $4 \mathrm{~d} \mathrm{U}(1)$ gauge theory one needs to choose a $\mathrm{U}(1)$ current (which is a linear combination of $J_{l}$ with integer coefficients) and add to the action a boundary term

$$
\frac{i}{2 \pi} \int_{\partial M} A \sum_{l} \mathrm{v}_{l} d a^{l}
$$

The row-vector $\mathrm{v}_{l}$ is integral.

Ordinarily, one requires the matrix $\mathrm{k}_{j l}$ to be nondegenerate, so that after gauge-fixing there is a well-defined propagator. However, in the presence of a coupling to bulk fields one can relax this assumption. Indeed, if $\mathrm{k}$ is degenerate, this means that certain fields simply do not appear in the Chern-Simons part of the Lagrangian. However, the linear combination $\mathrm{v}_{l} a^{l}$ appears in the term (2.3). If the kernel of $\mathrm{k}$ is one-dimensional, and the row-vector $v$ does not annihilate it, then the field which does not appear in the ChernSimons part of the action is simply a Lagrange multiplier, integration over which forces an integer multiple of $A$ to be trivial on the boundary. For example, the PEC condition corresponds to $n=1, \mathrm{k}=0$, and $\mathrm{v}=1$. Thus we will assume that $\mathrm{k}$ is either nondegenerate, or has a one-dimensional kernel which is not annihilated by v. We will call such a boundary condition nondegenerate. If we regard $v$ as a linear operator $v: \mathbb{R}^{n} \mapsto \mathbb{R}$, then the nondegeneracy condition can be compactly written as ker $\mathrm{k} \cap$ ker $\mathrm{v}=0$.

Finally, one may also add to the boundary action a Chern-Simons term for the restriction of the bulk gauge field:

$$
\frac{i \mathrm{p}}{4 \pi} \int_{\partial M} A d A
$$

Gauge-invariance requires the coefficient $\mathrm{p}$ to be integral. The most general boundary condition we are going to consider is described by an integral square matrix $k$, an integral vector $v$ and an integer $p$. The $\operatorname{SL}(2, \mathbb{Z})$ duality group acts on this set of boundary conditions, as first explained in [14]. 
On the classical level, we can integrate out the fields $a_{l}$ and get an ordinary boundary condition for $A$. However, in general this leads to a boundary effective action for $A$ which looks like Chern-Simons action with a fractional coefficient. This is inconsistent on the quantum level.

In particular, this means that a consistent treatment of a "perfect dyonic conductor" boundary condition requires an introduction of topological degrees of freedom living on the boundary.

\section{2 $\mathrm{SL}(2, \mathbb{Z})$ action on boundary conditions}

It follows from the results of [14] that the set of boundary conditions introduced above is closed with respect to the action of the group of duality transformations. In this section we review how this comes about following the approach of [4]; in particular, we show that the moduli of PEC and PMC boundary conditions are mapped into each other by electric-magnetic duality.

The duality group of the $\mathrm{U}(1)$ gauge theory is $\mathrm{SL}(2, \mathbb{Z})$. Its action on the parameters of the theory is most easily described by introducing

$$
\tau=\frac{\theta}{2 \pi}+\frac{2 \pi i}{e^{2}}
$$

Then an element of $\mathrm{SL}(2, \mathbb{Z})$

$$
g=\left(\begin{array}{ll}
a & b \\
c & d
\end{array}\right), \quad a, b, c, d \in \mathbb{Z}, \quad a d-b c=1
$$

acts on $\tau$ by

$$
\tau \mapsto \hat{\tau}=\frac{a \tau+b}{c \tau+d} .
$$

The same transformation maps a dyon of electric charge $n$ and magnetic charge $m$ to a dyon whose charges are

$$
\left(\begin{array}{l}
\hat{n} \\
\hat{m}
\end{array}\right)=g^{-1}\left(\begin{array}{c}
n \\
m
\end{array}\right) .
$$

As explained in [4], any element $g \in \mathrm{SL}(2, \mathbb{Z})$ gives rise to a defect of codimension 1 in the $4 \mathrm{~d}$ theory which separates two U(1) gauge theories related by a duality transformation. The action of $g$ on a boundary condition is obtained by fusing the defect with the boundary. For brevity we will call the nonlocal operator corresponding to a defect of codimension 1 the wall operator. Operators corresponding to defects of codimension 2 and 3 will be called surface operators and line operators, respectively. For example, the Wilson loop is a line operator.

The group $\mathrm{SL}(2, \mathbb{Z})$ is generated by elements

$$
T=\left(\begin{array}{ll}
1 & 1 \\
0 & 1
\end{array}\right), \quad S=\left(\begin{array}{cc}
0 & 1 \\
-1 & 0
\end{array}\right),
$$

so it is sufficient to determine how these elements act on boundary degrees of freedom. 
The $T$-transformation (i. e. the shift $\theta \rightarrow \theta+2 \pi$ ) is realized by the wall operator

$$
\exp \left(-\frac{i}{4 \pi} \int_{W} A d A\right)
$$

Here $W$ is a 3 -dimensional submanifold in $M$. This wall is topological because the expression (2.4) is independent of the metric, and therefore does not affect the stress-energy tensor. To illustrate that this is indeed the wall which corresponds to the $T$-transformation, consider its action on a 't Hooft loop operator localized on a circle $\gamma$ embedded into the interior of $M$. Let $W$ be a wall which is the boundary of a tubular neighborhood of $\gamma$. The 't Hooft operator is defined by the condition that the curvature $F$ is singular at $\gamma$ so that the integral of $F$ over a small 2 -sphere linking $\gamma$ is $2 \pi m$. Here $m$ is the magnetic charge of the 't Hooft loop operator. Then the wall operator becomes

$$
\exp \left(-i m \int_{\gamma} A\right)
$$

Thus the 't Hooft loop operator with magnetic charge $m$ is transformed into a Wilson't Hooft loop operator with electric charge $-m$ and magnetic charge $m$. This is the expected result.

Next we consider the wall operator implementing the $S$-transformation, i. e. the electric-magnetic duality. As explained in [4], it is defined as follows. Suppose $W$ splits $M$ into two disjoint pieces which we call $M_{-}$and $M_{+}$. We will choose the orientation of $W$ so that it agrees with the one induced from $M_{-}$and disagrees with the one induced from $M_{+}$. Let $A$ and $\hat{A}$ be the gauge fields living on $M_{-}$and $M_{+}$. We do not put any constraints on their restrictions to $W$ and add the following term to the action:

$$
\frac{i}{2 \pi} \int_{W} A d \hat{A}
$$

We denote the gauge couplings and theta-angles of the gauge theories on $M_{-}$and $M_{+}$by $e, \theta$ and $\hat{e}, \hat{\theta}$, respectively.

To see that this wall operator implements the $S$ transformation, we may do the following. Varying the action and requiring the part of the variation localized on $W$ to vanish, we find the matching of the fields across the boundary:

$$
\begin{aligned}
\left.\hat{F}\right|_{W} & =\left.\frac{2 \pi i}{e^{2}} * F\right|_{W}-\left.\frac{\theta}{2 \pi} F\right|_{W}, \\
\left.F\right|_{W} & =-\left.\frac{2 \pi i}{\hat{e}^{2}} * \hat{F}\right|_{W}+\left.\frac{\hat{\theta}}{2 \pi} \hat{F}\right|_{W} .
\end{aligned}
$$

Even if we do not impose any relation between $e, \theta$ and $\hat{e}, \hat{\theta}$, these formulas define a wall between the two gauge theories. However, this wall will not be a topological wall, in general. For a topological wall, all components of the stress-energy tensors $T$ and $\hat{T}$ must match on $W$. The stress-energy tensor is given by

$$
T_{\mu \nu}=\frac{1}{e^{2}}\left(F_{\mu \alpha} F_{\nu}^{\alpha}+\frac{1}{4} g_{\mu \nu} F_{\alpha \beta} F^{\alpha \beta}\right) .
$$


Using the matching conditions (2.5) one can easily check that $T=\hat{T}$ on the wall if and only if one has the relationship

$$
\hat{\tau}=-1 / \tau,
$$

as expected.

Now let us describe the action of $S$ and $T$ wall operators on boundary conditions. When the $T$-wall merges with a boundary, the boundary action is modified by a term

$$
\frac{i}{4 \pi} \int_{\partial M} A d A
$$

This is the $T$ transformation as defined in [14]. Thus the matrix $\mathrm{k}$ and the row-vector $\mathrm{v}$ are unchanged, while the integer $\mathrm{p}$ is shifted:

$$
\mathrm{k} \mapsto \mathrm{k}, \quad \mathrm{v} \mapsto \mathrm{v}, \quad \mathrm{p} \mapsto \mathrm{p}+1
$$

The effect of the $S$-transformation is more interesting. We assume that the wall is oriented so that $M_{-}$is the gap between the boundary and the wall. Then in the limit when the gap disappears $A$ becomes a new boundary gauge field. The bulk action for $A$ can be dropped in this limit, and the new boundary action is

$$
\sum_{j l} \frac{i \mathrm{k}_{j l}}{4 \pi} \int_{\partial M} a^{j} d a^{l}+\frac{i}{2 \pi} \int_{\partial M} a^{n+1} \sum_{l=1}^{n} \mathrm{v}_{l} d a^{l}+\frac{i}{2 \pi} \int_{\partial M} a^{n+1} d \hat{A}
$$

where we renamed $A=a^{n+1}$. One can describe the effect of the $S$ transformation by saying that the external gauge field $A$ has been replaced by a boundary gauge field $a^{n+1}$, and the new external gauge field $A=A_{+}$is coupled to the boundary current

$$
J^{n+1}=\frac{1}{2 \pi} d a^{n+1} .
$$

This is precisely the $S$ transformation as defined in [14]. It increases the number of the boundary fields by one and transforms the data $(k, v, p)$ as follows:

$$
\left\{\begin{array}{l}
\mathrm{k} \mapsto \tilde{\mathrm{k}}=\left(\begin{array}{ll}
\mathrm{k} & \mathrm{v}^{t} \\
\mathrm{v} & \mathrm{p}
\end{array}\right), \\
\mathrm{v} \mapsto \tilde{\mathrm{v}}=(0, \ldots, 0,1), \\
\mathrm{p} \mapsto \tilde{\mathrm{p}}=0 .
\end{array}\right.
$$

It is easy to see that the new boundary condition is non-degenerate in the sense defined above, i.e. that if $\operatorname{ker} \mathrm{k} \cap \operatorname{ker} v=0$, then $\operatorname{ker} \tilde{\mathrm{k}} \cap \operatorname{ker} \tilde{\mathrm{v}}=0$. Indeed, any $\tilde{u}$ that lies in $\operatorname{ker} \tilde{\mathrm{v}}$ is of the form $\left(\begin{array}{c}\vec{u} \\ 0\end{array}\right)$ for an arbitrary $n$-vector $\vec{u}$. But then

$$
\tilde{\mathrm{k}} \cdot \tilde{u}=\left(\begin{array}{c}
\mathrm{k} \vec{u} \\
\overrightarrow{\mathrm{v}} \cdot \vec{u}
\end{array}\right)=0 \quad \Leftrightarrow \quad \vec{u}=0,
$$

which is what we had to show. 
To illustrate this general procedure of dualizing boundary conditions, let us show that PEC and PMC boundary conditions are indeed dual to each other. The PEC boundary condition can be viewed as a special case where $n=1, \mathrm{k}=0$, and the boundary action is

$$
\frac{i}{2 \pi} \int_{\partial M} A d a^{1}
$$

Integration over $a^{1}$ yields a gauge-invariant delta-functional which sets $A$ to the trivial connection. $S$ transformation maps it to

$$
\frac{i}{2 \pi} \int_{\partial M} a^{2} d a^{1}+\frac{i}{2 \pi} \int_{\partial M} a^{2} d A
$$

Integration over $a^{2}$ gives a delta-functional which sets $a^{1}=-A$. Integration over $a^{1}$ then gives a theory with zero boundary action and no boundary fields. This is the PMC boundary condition.

The PMC boundary condition is a special case with $n=0$ and trivial boundary action. The $S$ transformation introduces a single boundary gauge field $a^{1}$ which couples to $A$ via

$$
\frac{i}{2 \pi} \int_{\partial M} a^{1} d A
$$

This is precisely the PEC boundary condition.

In the same way we can show that $S$ duality maps the moduli of the PMC boundary condition to the moduli of the PEC boundary condition. For example, the $S$ transformation maps the generalized PMC boundary condition to

$$
\frac{i}{2 \pi} \int_{\partial M}\left(a^{1} d A+a^{1} d \lambda\right)
$$

Integration over $a^{1}$ then gives a delta-functional which sets $A=-\lambda$ up to a gauge transformation. This is the generalized PEC boundary condition.

Applying more general elements of $\mathrm{SL}(2, \mathbb{Z})$ to the PEC and PMC boundary conditions we get boundary conditions corresponding to "perfect dyonic conductors" with dyons having relatively-prime electric and magnetic charges $(n, m)$. For example, applying the transformation $T^{k}$ to the PMC boundary condition, we find that a dyonic superconductor with dyons of charge $(-k, 1)$ can be modeled by a boundary Chern-Simons term at level $k$. Applying to this boundary condition the transformation $S^{-1}$, we find the boundary condition for the dyonic superconductor with dyons of charge $(1, k)$. It involves a boundary gauge field $a$ with an action

$$
\frac{i k}{4 \pi} \int_{\partial M} a d a-\frac{i}{2 \pi} \int_{\partial M} a d A
$$

\subsection{The action of duality on operators}

We can use the $S$-wall to determine the action of electric-magnetic duality on various operators, both local and nonlocal. Let us give some examples. 


\subsubsection{Wilson and 't Hooft loops}

We have already shown that the $S$-transformation maps the 't Hooft loop operator to a Wilson loop operator. Now let us show that the $S$-transformation maps the Wilson loop operator to a 't Hooft loop operator. Consider the Wilson operator

$$
W_{n}(\gamma)=\exp \left(\operatorname{in} \int_{\gamma} A\right)
$$

where $\gamma$ is a circle embedded into $M$, and $n \in \mathbb{Z}$ is the electric charge. Here is an outline of the argument that will allow us to calculate its dual: we begin by regularizing this operator and introducing a parameter $\epsilon$. We will then show that in the dual theory, only specific topological sectors contribute to the path integral, separate the contribution of the topological representative and of the topologically trivial part of the dual field, and find that this will allow us to calculate the result of sending $\epsilon$ to zero.

Let $\mathcal{Z}_{\gamma}^{\epsilon}$ be a tubular neighborhood of $\gamma$, with $\epsilon$ being its "thickness". To regularize the Wilson operator, we will need a closed and coclosed two-form $\Omega_{n}$ on $M \backslash \mathcal{Z}_{\gamma}^{\epsilon}$ whose periods are integral multiples of $2 \pi$ and which satisfies the following two conditions. First, it must satisfy

$$
\left.* \Omega_{n}\right|_{\partial \mathcal{Z}_{\gamma}^{\epsilon}}=0 .
$$

To explain the second condition, let us identify $\mathcal{Z}_{\gamma}^{\epsilon}$ with $S^{1} \times B_{\epsilon}^{3}$, where $B_{\epsilon}^{3} \subset \mathbb{R}^{3}$ is a ball of radius $\epsilon$. It is assumed that $\gamma$ has the form $S^{1} \times\{0\}$, where $\{0\}$ is the center of $B_{\epsilon}^{3}$. For any $p \in S^{1}$ we may consider the 2 -sphere $S_{\gamma}^{2}=\{p\} \times \partial B^{3}$ as a submanifold of $M$. Clearly, all such 2-spheres are homologous, so the integral of $\Omega_{n}$ over $S_{\gamma}^{2}$ does not depend on the choice of $p \in S^{1}$. We require the integral to be equal to $2 \pi n$.

If $\gamma$ is homologically trivial, then one can define an integer-valued linking number between $\gamma$ and any 2-cycle $c$ in the complement of $\gamma$. This is done as follows: if $D$ is a 2chain whose boundary is $\gamma$, then the linking number is the algebraic number of intersection points of $D$ and $c$. One can show that this number does not depend on the choice of $D$ and does not change if one replaces $c$ by a homologous 2-cycle. It is easy to see that $\gamma$ and $S_{\gamma}^{2}$ have linking number one, which means that $S_{\gamma}^{2}$ is homologically nontrivial when regarded as a cycle in $M \backslash \gamma$.

Let us show that the form $\Omega_{n}$ indeed exists, beginning with the case when the loop $\gamma$ is homologically trivial. The question of existence of a harmonic representation of a de Rham cohomology class on a manifold with boundary is classical and is discussed for example in $[6,7]$ (see also [15]); in particular, one result is that if we define "absolute" boundary conditions for a form $\alpha$ as $\left.* \alpha\right|_{\partial M}=0,\left.* d \alpha\right|_{\partial M}=0$, one has the isomorphism $H^{p}(M, \mathbb{R}) \simeq$ $\operatorname{Harm}_{\text {abs }}^{p}(M)$. This follows from the following analog of the Hodge decomposition theorem on a manifold with boundary:

$$
\Omega^{p}(M)=d \Omega^{p-1}(M) \oplus d^{*} \Omega_{\mathrm{tan}}^{p+1}(M) \oplus \operatorname{Harm}_{\mathrm{abs}}^{p}(M),
$$

where $\Omega_{\tan }$ denotes forms satisfying the "tangential" boundary condition $\left.* \alpha\right|_{\partial M}=0$.

This result guarantees the existence of a harmonic representative in each absolute cohomology class. A suitable cohomology class, however, exists only under a certain condition. 
In the case of a single Wilson operator, this condition is the requirement that $S_{\gamma}^{2}$ should not be contractible in $M \backslash \mathcal{Z}_{\epsilon}$, otherwise the constraint $\int_{S_{\gamma}^{2}} \Omega_{n}=2 \pi n$ would contradict the fact that $\Omega_{n}$ is closed. This condition is satisfied if the linking number of $S_{\gamma}^{2}$ and $\gamma$ is one.

We may consider a more general case when several Wilson loops $\gamma_{1}, \ldots, \gamma_{K}$ with charges $n_{1}, \ldots, n_{K}$ are present simultaneously. We claim that a form $\Omega_{\left\{n_{i}\right\}}$ that integrates to $n_{i}$ on the 2-spheres $S_{\gamma_{i}}^{2}$ exists if and only if

$$
\sum_{i} n_{i}\left[\gamma_{i}\right]=0
$$

where $\left[\gamma_{i}\right]$ denotes the homology class of $\gamma_{i}$. Only in this case will we be able to find the $S$ dual operator corresponding to such a combination of Wilson loops. This, however, is not a problem since if this homological condition is not satisfied, the path integral calculating the corresponding Wilson loop correlator vanishes. (The action and the integration measure are invariant under shifts of the gauge field by a flat connection, so any operator insertion must either be invariant as well, or yield a zero correlator. The invariance condition is precisely (2.8)).

Let us now prove the statement we made about the existence of $\Omega_{\left\{n_{i}\right\}}$. If the topological condition on $\left\{\gamma_{i}\right\}$ is satisfied, there exists a 2-chain $D$ whose boundary is $n_{1}$ copies of $\gamma_{1}, n_{2}$ copies of $\gamma_{2}$, etc. It is therefore a relative 2-cycle in what we will call the "bulk": $M \backslash\left(\bigcup_{i} \mathcal{Z}_{\gamma_{i}}^{\epsilon}\right)$. Consider the Poincare-dual class in the absolute cohomology of the bulk. This Poincare-dual class has a harmonic de Rham representative with the desired boundary conditions (by the above-mentioned Hodge decomposition theorem on a manifold with boundary). This is the form $\Omega_{\left\{n_{i}\right\}}$ we need. Although we do not really need the converse statement, let us sketch its proof. If $\Omega_{\left\{n_{i}\right\}}$ exists, then it defines an absolute de Rham cohomology class whose Poincare-dual relative homology class can be realized by a 2-chain whose boundary lies on $\partial \bigcup_{i} \mathcal{Z}_{\gamma_{i}}^{\epsilon}$. It is easy to see that in fact this boundary is homologous to the sum

$$
\sum_{i} n_{i}\left[\gamma_{i}\right]
$$

Applying the long exact sequence of a pair, we conclude that this sum must be homologically trivial when regarded as a class in the bulk.

From now on, to simplify the notation, we will return to the case of a single Wilson loop $\gamma$; the generalization of the argument to multiple loops is straightforward. Having found $\Omega_{n}$, we define the regularized Wilson loop by

$$
W_{n}^{\epsilon}(\gamma)=\exp \left(\frac{i}{2 \pi} \int_{\partial \mathcal{Z}_{\epsilon}} A \wedge \Omega_{n}\right)
$$

To see that this is indeed a regularization of the Wilson loop, note that in the limit $\epsilon \rightarrow 0$ we may approximate $\gamma$ by a straight line in $\mathbb{R}^{4}$, and then in spherical coordinates $(t, r, \theta, \phi)$ with $t$ running along $\gamma$ the form $\Omega_{n}$ is well approximated by $2 \pi n$ times the unit volume form $\Omega$ on the two-sphere $\left(t=t_{0}, r=\epsilon\right)$. Then the integral in the exponential becomes

$$
\text { in } \int_{r=\epsilon} A_{t} d \Omega d t
$$


which is obviously a regularization of $\left(\right.$ in $\left.\int_{\gamma} A\right)$.

Having regularized the Wilson loop, we place the duality wall on the boundary of $\mathcal{Z}_{\epsilon}$; we will call $B$ the dual gauge field. Then the Wilson loop and the wall operator combine into a factor

$$
\exp \left(-\frac{i}{2 \pi} \int_{\partial \mathcal{Z}_{\epsilon}} A\left(d B-\Omega_{n}\right)\right)
$$

At this point a remark is in order. The 2 -form $\Omega_{n}$ may not be unique. This is easy to see from the way it was constructed above: the relative 2 -cycle $D$ is defined modulo an addition of an absolute 2-cycle. We may fix this ambiguity by requiring the flux of $\Omega_{n}$ through an absolute cycle $c_{i}$ to be $2 \pi m_{i}$ for some integer $m_{i}$. What does this freedom correspond to? Examining the boundary conditions associated with the boundary action (2.9), one can easily check that a saddle point for such a theory — or, equivalently, a solution to the classical field equations - exists only if $\int_{S_{\gamma}^{2}} d B=2 \pi n$, i.e. if its flux through $S_{\gamma}^{2}$ equals that of $\Omega_{n}$. (One obtains this by integrating the boundary conditions over $S_{\gamma}^{2}$.) This means that topological sectors not satisfying this flux condition do not contribute to the path integral. In each topological sector that does contribute, the curvature of $B$ can be written as

$$
F_{B}=\Omega_{n}+d B^{\prime}
$$

Here $B^{\prime}$ is a connection such that $d B^{\prime}$ has vanishing flux through $S_{\gamma}^{2}$, and $\Omega_{n}$ may be regarded as the curvature of some connection on a fixed $\mathrm{U}(1)$ bundle. Thus the ambiguity in the choice of $\Omega_{n}$ can be regarded as an ambiguity in the decomposition of $d B$ into a classical background $\Omega_{n}$ and a fluctuating part $d B^{\prime}$. Instead of fixing $\Omega_{n}$, one might sum over all possible fluxes of $\Omega_{n}$ through absolute cycles; then $B^{\prime}$ is a connection on a trivial $\mathrm{U}(1)$ bundle.

We now arrive at the final stage of the argument. Choosing in each topological sector an appropriate $\Omega_{n}$ in the sense just described, we make use of (2.10) and shift the integration variable in the path-integral:

$$
B=B^{\prime}+B_{n},
$$

where $B_{n}$ is some connection such that $d B_{n}=\Omega_{n}$. In terms of $B^{\prime}$ the wall operator (2.9) becomes simply

$$
\exp \left(-\frac{i}{2 \pi} \int_{W} A \wedge d B^{\prime}\right)
$$

The action then splits into parts involving only $B^{\prime}$ and only $B_{n}$ :

$$
S[B ; \tilde{e}, \tilde{\theta}]=S\left[B^{\prime} ; \tilde{e}, \tilde{\theta}\right]+S\left[B_{n} ; \tilde{e}, \tilde{\theta}\right],
$$

where $\tilde{e}, \tilde{\theta}$ are the coupling constants of the dual theory. Indeed, the Maxwell part of the action splits because $d B_{n}=\Omega_{n}$ is harmonic, and the $\theta$-term does not see the topologically trivial field $B^{\prime}$ :

$$
\int d\left(B_{n}+B^{\prime}\right) \wedge d\left(B_{n}+B^{\prime}\right)=\int d B_{n} \wedge d B_{n}
$$

In (2.11), the second term does not depend on $B^{\prime}$ and provides an overall factor in the path integral. Apart from this factor the path integral now has exactly the same form as 
in the absence of a Wilson loop, i. e. it has a duality wall inserted at $\partial \mathcal{Z}_{\epsilon}$ and nothing else. Sending $\epsilon$ to zero we are left with a path-integral for $B^{\prime}$ with the standard Maxwell action $S\left[B^{\prime} ; \tilde{e}, \tilde{\theta}\right]$. There is also an overall factor

$$
\exp \left(-S\left[B_{n} ; \tilde{e}, \tilde{\theta}\right]\right)=\exp \left(-\frac{1}{2 \tilde{e}^{2}} \int_{M} d B_{n} \wedge * d B_{n}-\frac{i \tilde{\theta}}{8 \pi^{2}} \int_{M} d B_{n} \wedge d B_{n}\right)
$$

Recombining it with the action for $B^{\prime}$ we get the expected action for a field composed of a topologically trivial part $B^{\prime}$ and a topological representative $B_{n}$ with a singularity on $\gamma$ :

$$
S\left[B_{n}+B^{\prime} ; \tilde{e}, \tilde{\theta}\right] .
$$

We may therefore say that the dual of $W_{n}$ is the prescription to integrate over all fields $B$ that are singular at $\gamma$ so that the integral of their curvature over the sphere $S_{\gamma}^{2}$ equals $2 \pi n$. This is the 't Hooft line operator in the theory of the field $B$.

\subsubsection{Chern-Simons operator}

Another interesting nonlocal operator is the Chern-Simons operator

$$
\exp \left(-\frac{i k}{4 \pi} \int_{W} A d A\right)
$$

It is an operator localized at an oriented connected 3-manifold $W \subset M$. In the traditional approach, it is not obvious how to find the $S$-dual of such a nonlocal operator, because it cannot be written as a function of $F=d A$ alone.

In the duality wall approach, we consider the tubular neighborhood of $W$, which is isomorphic to $W \times[-1,1]$, and place the $S$-wall at $W \times p_{1}$ and the parity-reversed $S$-wall at $W \times p_{-1}$, where $p_{1}$ and $p_{-1}$ are the right and left boundaries of the interval, respectively. Let us denote $W_{-}=W \times p_{-1}$ and $W_{+}=W \times p_{1}$. We may regard $W_{+}$and $W_{-}$as submanifolds of $M$ (the connected components of the boundary of the tubular neighborhood of $W$ ). We rename the gauge field living outside the tubular neighborhood $A$, and its restrictions to $W_{+}$and $W_{-}$will be denoted $A_{+}$and $A_{-}$, respectively. In the limit when the width of the tubular neighborhood goes to zero, the gauge field $a$ living on $W \times[-1,1]$ becomes three-dimensional, with an action

$$
\frac{i}{2 \pi} \int_{W}\left(\frac{k}{2} a d a+a d A_{+}-a d A_{-}\right)
$$

Thus the $S$-dual of the Chern-Simons operator localized on $W$ is a disorder operator defined by coupling the $\mathrm{U}(1)$ Chern-Simons theory on $W$ to the bulk fields in the way indicated in (2.12). The traditional approach to $S$-duality runs into trouble in the case of the ChernSimons operator because its dual involves a new field living on $W$.

Since the action for the wall gauge field $a$ is Gaussian, one could try to integrate it out by solving its equations of motion and substituting back into the action. The equation of motion reads

$$
k d a=d\left(A_{-}-A_{+}\right) .
$$


Naively, one could therefore replace $a$ with $\frac{1}{k}\left(A_{-}-A_{+}\right)$and get the following effective action:

$$
-\frac{i}{2 \pi} \int_{W} \frac{1}{2 k}\left(A_{+}-A_{-}\right) d\left(A_{+}-A_{-}\right) .
$$

This is the Chern-Simons action with fractional level $-1 / k$. On the quantum level this is problematic because the resulting wall operator is not invariant under large gauge transformation. To avoid this difficulty, one has to keep the wall field $a$, even though it only has topological degrees of freedom.

Note that the Chern-Simons operator is nothing but the wall operator corresponding to the duality transformation

$$
T^{k}=\left(\begin{array}{ll}
1 & k \\
0 & 1
\end{array}\right) .
$$

Its $S$-dual is the wall operator corresponding to the transformation

$$
S^{-1} T^{k} S=\left(\begin{array}{cc}
1 & 0 \\
-k & 1
\end{array}\right)
$$

\section{Abelian duality in $2 \mathrm{~d}$}

\section{$3.1 \quad$ Toroidal sigma-model}

The $2 \mathrm{~d}$ field theory we are going to study is the sigma-model whose target is a torus $X=T^{N} \simeq \mathbb{R}^{N} / \mathbb{Z}^{N}$ with a flat metric $g$ and a constant B-field $B \in \Omega^{2}(X)$. The map from the worldsheet $M$ to $X$ can be thought of as an $N$-component scalar field $\Phi^{I}, I=1, \ldots, N$ with identifications $\Phi^{I} \simeq \Phi^{I}+2 \pi v^{I}$, where $v^{I}$ is an arbitrary element of $\mathbb{Z}^{N}$. The Euclidean action is

$$
S=\frac{1}{4 \pi} \int_{M}\left(g_{I J} d \Phi^{I} \wedge * d \Phi^{J}+i B_{I J} d \Phi^{I} \wedge d \Phi^{J}\right) .
$$

This class of sigma-models is known to be acted upon by the duality group $S p(2 n, \mathbb{Z})$. Theories related by this action are equivalent on the quantum level. The most obvious duality transformation is a shift of a B-field:

$$
B \mapsto B+8 \pi^{2} \beta
$$

where $\beta$ is any 2 -form on $X$ with integral periods. Such a shift modifies the action by a topological term which is an integer times $2 \pi i$, and therefore does not affect the pathintegral. B-field shifts are analogous to shifts of the theta-angle in the $4 \mathrm{~d}$ gauge theory.

Another class of duality transformations is T-duality. We will only consider the simplest T-duality which replaces $X$ with the dual torus $\hat{X}$. The metric and the B-field on $\hat{X}$ are given by

$$
\hat{g}+\hat{B}=(g+B)^{-1} .
$$

We will call this the basic T-duality. More general T-dualities correspond to decomposing $X$ into a product of two tori and dualizing one of the factors. 
First let us exhibit the duality wall corresponding to the B-field shift. It is a line operator supported on a one-dimensional submanifold $\Gamma \in M$ and given by

$$
\exp \left(-2 \pi i \int_{\Gamma} \beta_{I J} \Phi^{I} d \Phi^{J}\right)
$$

Since the fields $\Phi^{I}$ are defined only up to integer multiples of $2 \pi$, care is required in interpreting this expression. By analogy with the usual Chern-Simons action, one could try to define it by picking a Riemann surface $\Sigma$ with $\partial \Sigma=\Gamma$, extending the map $\Phi: \partial \Sigma \rightarrow X$ to a map $\tilde{\Phi}: \Sigma \rightarrow X$, and defining the formal expression (3.2) as

$$
\exp \left(-i \int_{\Sigma} \tilde{\Phi}^{*} \beta\right)
$$

It is easy to see that it does not depend on the choice of $\Sigma$ and $\tilde{\Phi}$. However, unlike the case of vector bundles, here an extension $(\Sigma, \tilde{\Phi})$ exists only under a very restrictive condition: the homology class $\Phi_{*}(\Gamma) \in H_{1}(X)$ must be trivial. Since $X$ is a torus, if this condition is satisfied, $\Phi$ can be lifted to a map $\Gamma \rightarrow \mathbb{R}^{N}$, and there is no difficulty in interpreting the expression (3.2) anyway.

A better approach is to regard $\beta$ as the curvature 2 -form of a unitary connection $\nabla_{\beta}$ on a line bundle on $X$. Then one can define (3.2) as the holonomy of the pull-back connection $\Phi^{*} \nabla_{\beta}$. The resulting wall operator is well-defined, but it depends on the choice of $\nabla_{\beta}$, not just on $\beta$. This dependence on $\nabla_{\beta}$ will show up when we consider the action of the duality wall on boundary conditions.

Note that if $\Gamma$ separates the worldsheet $M$ into two disconnected domains, and there are no disorder operator insertions on one or both of these domains, the map $\Phi$ is necessarily homologically trivial, and the definition using the holonomy of a connection reduces to the naive one.

The duality wall corresponding to the basic T-duality is a disorder line operator separating parts of the worldsheet $M$ where the sigma-models with targets $X$ and $\hat{X}$ live. Let $\hat{\Phi}_{I}$ be the fields dual to $\Phi^{I}$. Then the wall corresponding to the basic T-duality is

$$
\exp \left(-\frac{i}{2 \pi} \int_{\Gamma} \hat{\Phi}_{I} d \Phi^{I}\right)
$$

Again some care is needed in interpreting this expression. Recall that $\hat{X}$ is, by definition, the moduli space of flat $\mathrm{U}(1)$ connections on $X$, and that the connection corresponding to a point $p=\left(\hat{\Phi}^{1}, \ldots, \hat{\Phi}^{N}\right) \in \hat{X}$ is

$$
\frac{1}{2 \pi} \hat{\Phi}_{I} d \Phi^{I}
$$

One can regard this connection on $X$ as the restriction of the canonical connection on the Poincaré line bundle on $X \times \hat{X}$ to $X \times\{\hat{p}\}$, where $\hat{p}$ has coordinates $\hat{\Phi}_{J}$. The curvature of this connection is

$$
-\frac{1}{2 \pi} d \Phi^{I} \wedge d \hat{\Phi}_{I} .
$$

Then it is natural to define the T-duality wall as the holonomy of the pull-back of the connection on the Poincaré line bundle with respect to the map $(\Phi, \hat{\Phi}): \Gamma \rightarrow X \times \hat{X}$. 
Varying the action in the presence of the T-duality wall and requiring the vanishing of the boundary terms we get the following matching conditions on $\Gamma$ :

$$
\begin{aligned}
& \left.\left(g_{I J} * d \Phi^{J}+i B_{I J} d \Phi^{J}+i d \hat{\Phi}_{I}\right)\right|_{\Gamma}=0 \\
& \left.\left(\hat{g}^{I J} * d \hat{\Phi}_{J}+i \hat{B}^{I J} d \hat{\Phi}_{J}+i d \Phi^{I}\right)\right|_{\Gamma}=0
\end{aligned}
$$

Requiring that the stress-energy tensors match on $\Gamma$ then gives the relation (3.1) between $\hat{g}, \hat{B}$ and $g, B$. To see this, we introduce the notations $\sigma^{0,1}$ and $h_{\alpha \beta}$ for the local coordinates and metric on $M$, denote $F_{\alpha}^{I} \equiv \frac{\partial \Phi^{I}}{\partial \sigma^{\alpha}}$ and write the stress-energy tensor as

$$
T^{\alpha \beta}=\frac{1}{4 \pi} g_{I J} F_{\gamma}^{I} F_{\delta}^{J}\left(\frac{1}{2} h^{\gamma \delta} h^{\alpha \beta}-h^{\alpha \gamma} h^{\beta \delta}\right)
$$

To simplify the calculations, note that the metric $h^{i j}$ can be turned into $\delta^{i j}$ by a local diffeomorphism and that without loss of generality we can assume the "wall" $\Gamma$ to be locally given by the equation $\sigma^{1}=0$. We can then use the matching conditions (3.4) to express $\hat{F}_{I}$ through $F^{J}$ at the wall and verify that of the two independent components of $T^{\alpha \beta}, T_{1}^{0}=\hat{T}_{1}^{0}$ identically, while requiring $T_{0}^{0}=\hat{T}_{0}^{0}$ gives the condition (3.1).

\subsection{Boundary conditions for the sigma-model}

A natural class of boundary conditions for the toroidal sigma-model is written by analogy with the $4 \mathrm{~d}$ case. We introduce $n$ boundary scalars $\phi^{i}, i=1, \ldots, n$, with identifications $\phi^{i} \sim \phi^{i}+2 \pi v^{i}, v \in \mathbb{Z}^{n}$, and an action

$$
S_{b}=\frac{i}{2 \pi} \int_{\gamma}\left[\frac{1}{2} \kappa_{j k} \phi^{j} d \phi^{k}+\rho_{J j} \Phi^{J} d \phi^{j}+\frac{1}{2} \nu_{J K} \Phi^{J} d \Phi^{K}\right],
$$

where $\gamma$ is a connected component of $\partial M$. Unlike in $4 \mathrm{~d}$, here the matrices $\kappa_{i j}$ and $\nu_{J K}$ are anti-symmetric rather than symmetric. For the boundary theory to be well-defined, $\kappa$ has to be nondegenerate, and then we may regard it as a symplectic form on the torus $Y \simeq \mathbb{R}^{n} / \mathbb{Z}^{n}$ parameterized by $\phi^{i}$. Later we will relax the nondegeneracy constraint.

Since $\phi^{i}$ are defined up to addition of integer multiples of $2 \pi$, the expression (3.5) requires an interpretation. As above, we introduce a unitary line bundle $\mathcal{L}$ on $Y \times X$ and equip it with a connection $\nabla$ whose curvature is

$$
\frac{1}{2 \pi}\left(\frac{1}{2} \kappa_{j k} d \phi^{j} d \phi^{k}+\rho_{J j} d \Phi^{J} d \phi^{j}+\frac{1}{2} \nu_{J K} d \Phi^{J} d \Phi^{K}\right)=\frac{1}{2 \pi}(\kappa+\rho+\nu) .
$$

For this to make sense, the periods of the curvature 2 -form must be $2 \pi$ times an integer, and this requires the matrices $\kappa, \rho, \nu$ to be integral. The choice of $\kappa, \rho, \nu$ determines the topology of the line bundle $\mathcal{L}$, but leaves some freedom in the choice of $\nabla$. Any two connections with curvature (3.6) differ by a flat connection.

In the presence of the boundary-bulk coupling the nondegeneracy constraint on $\kappa$ can be relaxed. Indeed, if the boundary fields in the kernel of $\kappa$ all couple nontrivially to the bulk fields $\Phi^{J}$, then they can be regarded as Lagrange multiplier fields whose equations of motion enforce Dirichlet boundary conditions for some linear combinations of $\Phi^{J}$. In other 
words, the boundary condition is well-defined if $\rho$, regarded as a map from $\mathbb{R}^{n}$ to $\mathbb{R}^{N}$, is injective when restricted to the kernel of $\kappa$ : $(\operatorname{ker} \kappa) \cap(\operatorname{ker} \rho)=0$. A boundary condition satisfying this constraint will be called nondegenerate.

\subsection{D-brane interpretation}

Let us now explain the interpretation of this class of boundary conditions in terms of Dbranes. Let us first assume that $\kappa$ is nondegenerate. The first step is to quantize the boundary degrees of freedom regarding the bulk fields $\Phi^{J}$ as a classical background. That is, we fix a point $p \in X$ and take $\Phi^{J}$ to be the coordinates of this point. Quantization of the boundary fields $\phi^{j}$ is a standard exercise in geometric quantization. The torus $Y$ carries a symplectic form $\kappa$ whose periods are integral multiples of $(2 \pi)^{2}$. Thus there exists a unitary line bundle $\mathcal{L}_{p}$ on $Y$ with a connection $\nabla_{p}$ whose curvature is $\kappa /(2 \pi)$. While the topology of the line bundle $\mathcal{L}_{p}$ is determined by $\kappa$, the connection is defined only up to addition of a closed 1-form. Thus we have to make a choice. This is the same choice we had to make above when giving the precise meaning to $\exp \left(-S_{b}\right)$. The bundle $\mathcal{L}_{p}$ and the connection $\nabla_{p}$ are simply the restrictions of $\mathcal{L}$ and $\nabla$ to the submanifold $Y \times\{p\}$.

To perform quantization, one also needs to choose a complex structure on $Y$ so that $\kappa$ is a Kähler form, i. e. of type $(1,1)$ and positive. This is always possible to do. Then the quantum-mechanical Hilbert space is the space of holomorphic sections of $\mathcal{L}_{p}$. Its dimension is given by the Riemann-Roch formula:

$$
h^{0}\left(Y, \mathcal{L}_{p}\right)=\int_{Y} \exp \left(\frac{\kappa}{(2 \pi)^{2}}\right)=\operatorname{Pf}(\kappa) .
$$

From the point of view of D-branes, this means that we have a D-brane on $X$ of $\operatorname{rank} \operatorname{Pf}(\kappa)$.

It is helpful to think about the problem of quantization of the periodic scalars $\phi^{j}$ in more physical terms. Consider a nonrelativistic charged spinless particle on the torus $Y$. We assume that there is electromagnetic field on $Y$ whose field-strength is constant and given by $\frac{1}{2 \pi} \kappa$. In this situation the spectrum of the Hamiltonian is discrete; the energy eigenspaces are called Landau levels. In the limit when the mass of the particle goes to infinity, the spacing between Landau levels becomes infinite, while the classical action describing the particle reduces precisely to (3.5). Thus the quantum theory corresponding to the action (3.5) describes the lowest Landau level. Holomorphic sections of the line bundle $\mathcal{L}_{p}$ are the wavefunctions for the lowest Landau level. Note that in the case when $\operatorname{dim} Y=2$, the formula for $h^{0}\left(Y, \mathcal{L}_{p}\right)$ reduces to

$$
h^{0}\left(Y, \mathcal{L}_{p}\right)=\kappa_{12}
$$

This is the familiar statement that the degeneracy of the Landau level is proportional to the magnetic field.

As one varies $p \in X$, the connection $\nabla_{p}$ changes, but the dimension of $H^{0}(Y, \mathcal{L})$ does not. In this way we obtain a vector bundle $E$ over $X$. It carries a natural connection: the Berry connection $[1,13]$. In mathematical terms, the Berry connection on $E$ arises as follows. $E$ is a subbundle of the infinite-dimensional bundle $\mathcal{E}$ over $X$ whose fiber over 
$p \in X$ is the space of all smooth sections of $\mathcal{L}_{p}$. The connection $\nabla$ on $\mathcal{L}$ gives rise to a connection on $\mathcal{E}$. This in turn induces a connection on the subbundle $E$ via the orthogonal projection. The evolution operator on the boundary Hilbert space is simply the holonomy of the Berry connection.

By definition, the charge of a D-brane corresponding to a vector bundle $E$ on $X$ is the Chern character of $E$. To compute it, note that the space of holomorphic sections of a positive line bundle on the complex torus $Y$ can be identified with the kernel of the Dirac operator on $Y$ twisted by this line bundle. Thus the Chern character of $E$ can be computed from the family version of the Atiyah-Singer index theorem:

$$
\operatorname{ch}(E)=\int_{Y} \exp \left(\frac{F}{2 \pi}\right)
$$

where the curvature $F$ is given by (3.6).

The simplest nontrivial example is $X=Y=T^{2}$. Then the matrices $\kappa$ and $\nu$ have the form

$$
\kappa_{j k}=\epsilon_{j k} \kappa_{0}, \quad \nu_{j k}=\epsilon_{j k} \nu_{0},
$$

where $\epsilon_{j k}$ is the antisymmetric tensor with $\epsilon_{12}=1$. The bundle $E$ has rank $\left|\kappa_{0}\right|$ and its Chern character is

$$
\operatorname{ch}(E)=\kappa_{0}+\frac{1}{(2 \pi)^{2}}\left(\kappa_{0} \nu_{0}-\operatorname{det} \rho\right) d \Phi^{1} d \Phi^{2},
$$

where $\operatorname{det} \rho \equiv \rho_{11} \rho_{22}-\rho_{12} \rho_{21}$

If $\kappa$ has a nontrivial kernel, the fields $\phi^{j}$ which parameterize the kernel are the Lagrange multiplier fields. Performing the path-integral over these fields puts linear constraints on the boundary values of the fields $\Phi^{J}$. These constraints are

$$
b_{\alpha}^{j} \rho_{J j} \Phi^{J}=0 \quad \bmod 2 \pi .
$$

where the columns of the integral matrix $\left\|b_{\alpha}^{j}\right\|$ generate the kernel of $\kappa$. The constraints define a linear submanifold $Z \subset X$, and quantization of the remaining fields $\phi^{j}$ yields a vector bundle $E$ over $Z$. The pair $(Z, E)$ defines a D-brane on $X$.

Let us compute the charge of this D-brane. By definition, it is a cohomology class on $X$ which is the push-forward of the Chern character of $E$ with respect to the embedding $Z \hookrightarrow X$. First let us assume that $\kappa=0$, so that all boundary gauge fields are the Lagrange multiplier fields. In this special case $Z$ is given by the equations

$$
\exp \left(i f_{j}\right)=1, \quad j=0, \ldots, n,
$$

where

$$
f_{j}=\rho_{J j} \Phi^{J}
$$

The bundle $E$ is the trivial bundle of rank one over $Z$. The charge is simply the Poincaré dual of the homology class of $Z$. Up to a sign, it can be represented by a distributional $n$-form

$$
\prod_{j=1}^{n} \tilde{\delta}\left(f_{j}\right) d f_{j}
$$


where we regard $f_{j}$ as a map from $X$ to $\mathbb{R} / 2 \pi \mathbb{Z}$ and $\tilde{\delta}(x)$ is a $\delta$-function on $\mathbb{R} / 2 \pi \mathbb{Z}$ concentrated at 0 . The precise sign depends on the choice of orientation for $Z$; we will fix it later.

We can smooth out the form (3.7) without changing its cohomology class by replacing the distribution $\tilde{\delta}$ with any function on $\mathbb{R} / 2 \pi \mathbb{Z}$ which integrates to 1 , such as the constant $\frac{1}{2 \pi}$. Then the smoothed-out form becomes

$$
(2 \pi)^{-n} d f_{1} \ldots d f_{n}
$$

Up to a sign, we can rewrite it in a more suggestive way as an integral over $Y$ of an inhomogeneous form on $Y \times X$ :

$$
\int_{Y} \exp \left(\frac{\rho}{(2 \pi)^{2}}\right)
$$

where

$$
\rho=d f_{j} d \phi^{j}=\rho_{J j} d \Phi^{J} d \phi^{j}
$$

and $\phi^{j}, j=1, \ldots, n$ are regarded as $2 \pi$-periodic coordinates on $Y$.

If $\kappa \neq 0, Y$ can be decomposed into a product of two tori, on the first of which $\kappa$ is nondegenerate, and the second one parameterized by Lagrange multiplier fields. Accordingly, the charge of the D-brane is a product of two contributions which can be computed as above. They nicely combine into a single integral over $Y$, so the final result for the charge of the D-brane is simply the cohomology class of the form

$$
\operatorname{ch}(Z, E)=\int_{Y} \exp \frac{\kappa+\rho+\nu}{(2 \pi)^{2}} .
$$

We implicitly fixed the orientation convention for $Z$ by requiring that this formula is valid without any additional sign factors whether $\kappa$ is nondegenerate or not.

\subsection{Duality action on boundary conditions}

Let us now consider the action of line operators representing dualities on boundary conditions we have introduced above. To this end one needs to merge the line operator representing the duality transformation with the boundary.

For the line operator corresponding to the shift of the B-field this is very simple: one simply adds to the boundary action a new term which depends only on the fields $\Phi^{J}$. As explained above, it is best to think about this line operator as the holonomy of a certain connection $\nabla_{\beta}$ on a line bundle $\mathcal{L}_{\beta}$ on $X$. Since the exponential of the boundary action is defined as the holonomy of a connection $\nabla$ on a line bundle on $Y \times X$, it is clear that the effect of the B-field shift is simply to add a new piece to the connection 1-form on $Y \times X$.

Adding a 1-form to a connection gives another connection on the same line bundle. In our case, we are adding not a globally-defined 1-form, but a pull-back to $Y \times X$ of a connection 1-form on the line bundle $\mathcal{L}_{\beta}$. The result is a connection on a line bundle on $Y \times X$ which is a product of $\mathcal{L}$ and the pull-back of $\mathcal{L}_{\beta}$. In other words, the effect of the B-field shift on the boundary condition is

$$
\mathcal{L} \mapsto \mathcal{L} \otimes \pi_{X}^{*} \mathcal{L}_{\beta},
$$


where $\pi_{X}: Y \times X \rightarrow X$ is the projection $(y, x) \mapsto x$.

Now let us consider the basic T-duality. In the limit when the disorder line operator (3.3) merges with the boundary, the fields $\Phi^{J}$ living in the gap between the boundary and the line operator become boundary fields. The new target space for the boundary degrees of freedom is $Y \times X$. The new boundary action is

$$
\frac{i}{2 \pi} \int_{\gamma}\left[\frac{1}{2} \kappa_{j l} \phi^{j} d \phi^{l}+\rho_{J j} \Phi^{J} d \phi^{j}+\frac{1}{2} \nu_{J K} \Phi^{J} d \Phi^{K}+\hat{\Phi}_{J} d \Phi^{J}\right] .
$$

This can be described in words as follows. Given a $2 \mathrm{~d}$ sigma-model with target $X$, the boundary condition is specified by a torus $Y$ and a unitary line bundle $\mathcal{L}$ on $Y \times X$ with a constant-curvature connection $\nabla$. The basic T-duality has the following effect:

$$
X \mapsto \hat{X}, \quad Y \mapsto Y \times X, \quad \mathcal{L} \mapsto \mathcal{L} \otimes \pi^{*} \mathcal{P},
$$

where $\mathcal{P}$ is the Poincaré line bundle on $X \times \hat{X}$ and $\pi$ is the projection $Y \times X \times \hat{X} \rightarrow X \times \hat{X}$.

These manipulations are similar to the Fourier-Mukai transform in algebraic geometry. There, one is given a pair of algebraic varieties $X$ and $\hat{X}$ as well as an object $B$ of the derived category of coherent sheaves on $X \times \hat{X}$. For simplicity one can think about the special case when $B$ is a holomorphic vector bundle on $X \times \hat{X}$. The object $B$ defines a functor from the derived category of $X$ denoted $D(X)$ to the derived category of $\hat{X}$ as follows. Given an object of $D(X)$ one pulls it back to $X \times \hat{X}$, tensors with $B$ and pushes forward (computes fiberwise cohomology) to $\hat{X}$. In our case, the role of objects of the derived category of $X$ is played by trivial torus fibrations over $X$ with line bundles on them. One can obviously tensor them over $X$. If the base of the fibration $X$ is decomposed into a product of two subtori $X_{1}$ and $X_{2}$, one can consider the projection map $\pi_{2}: X \rightarrow X_{2}$ and define the push-forward of a fibration $\mathcal{Y}$ over $X$ as the same fibration $\mathcal{Y}$ but regarded as a trivial torus fibration over $X_{2}$.

If the original boundary condition is nondegenerate, then so should be the T-dual boundary condition. To see this, we must check that the condition $(\operatorname{ker} \kappa) \cap(\operatorname{ker} \rho)=0$ is preserved under the transformation of the triplet $(\kappa, \rho, \nu)$ induced by $T$-duality (compare with (2.7)):

$$
\left\{\begin{array}{rlll}
\kappa_{n \times n} & \mapsto & \tilde{\kappa}_{(n+N) \times(n+N)} & =\left(\begin{array}{cc}
\kappa & \rho^{t} \\
\rho & \nu
\end{array}\right), \\
\rho_{N \times n} & \mapsto & \tilde{\rho}_{N \times(n+N)} & =\left(\mathbf{0}_{N \times n}, \mathbf{1}_{N \times N}\right), \\
\nu_{N \times N} & \mapsto & \tilde{\nu}_{N \times N} & =\mathbf{0}_{N \times N} .
\end{array}\right.
$$

Here the subscripts indicate the dimensions of the matrices. A vector $\tilde{u} \in \operatorname{ker} \tilde{\rho}$ is necessarily of the form $\left(\begin{array}{c}u_{n} \\ \mathbf{0}_{N}\end{array}\right)$, and we have

$$
\tilde{u} \in \operatorname{ker} \tilde{\kappa} \Leftrightarrow\left(\begin{array}{cc}
\kappa & \rho^{t} \\
\rho & \nu
\end{array}\right)\left(\begin{array}{l}
u \\
\mathbf{0}
\end{array}\right)=\left(\begin{array}{c}
\kappa u \\
\rho u
\end{array}\right)=0 \quad \Leftrightarrow \quad u=0, \quad \text { Q.E.D. }
$$

Thus the set of boundary conditions we have introduced is closed both with respect to the basic T-duality and the B-field shifts. 
Now it is easy to compute the action of the basic T-duality on D-brane charges. As explained above, the charge of a D-brane $(Z, E)$ corresponding to the triple $(X, Y, \mathcal{L})$ is the integral over $Y$ of $\operatorname{ch}(\mathcal{L})$. The charge of the T-dual D-brane is the integral of $\operatorname{ch}\left(\mathcal{L} \otimes \pi^{*} \mathcal{P}\right)$ over $Y \times X$. Since the Chern character is multiplicative with respect to the tensor product of line bundles, this is the same as the integral over $Y \times X$ of the product of $\operatorname{ch}(\mathcal{L})$ and $\pi^{*} \operatorname{ch}(\mathcal{P})$. But this is the same as the integral over $X$ of the product of $\operatorname{ch}(Z, E)$ and $\operatorname{ch}(\mathcal{P})$. Thus T-duality has the following effect on the D-brane charge:

$$
\operatorname{ch}(Z, E) \mapsto \operatorname{ch}(\hat{Z}, \hat{E})=\int_{X} \operatorname{ch}(Z, E) \operatorname{ch}(\mathcal{P}) .
$$

Note that this formula does not require us to know how to realize any particular D-brane charge on $X$ by a line bundle on $Y \times X$.

To illustrate this transformation law, let us consider a simple example with $n=2$, $\kappa_{i j}=r \epsilon_{i j}, \nu_{I J}=c \epsilon_{I J}$ and $\rho=0$. This corresponds to a D-brane on $T^{2}$ which has rank $r$ and first Chern class $c r$, and its charge is

$$
r+\frac{c r}{(2 \pi)^{2}} d \Phi^{1} d \Phi^{2}
$$

The T-dual boundary condition has $n=4, \hat{Y}=Y \times X$ and the line bundle $\hat{L}$ on $Y \times X \times \hat{X}$ whose first Chern class is

$$
\frac{1}{(2 \pi)^{2}}\left(r d \phi^{1} d \phi^{2}+c d \Phi^{1} d \Phi^{2}+d \hat{\Phi}_{I} d \Phi^{I}\right) .
$$

The charge of the corresponding (T-dual) brane on $\hat{X}$ can be computed by multiplying (3.8) by the Chern character of the Poincaré line bundle and integrating over $X$. The result is

$$
\int_{X}\left(r+c r \frac{d \Phi^{1}}{2 \pi} \frac{d \Phi^{2}}{2 \pi}\right)\left(1+\frac{d \hat{\Phi}_{I}}{2 \pi} \frac{d \Phi^{I}}{2 \pi}+\frac{1}{2}\left[\frac{d \hat{\Phi}_{I}}{2 \pi} \frac{d \Phi^{I}}{2 \pi}\right]^{2}\right)=c r-\frac{r}{(2 \pi)^{2}} d \hat{\Phi}_{1} d \hat{\Phi}_{2}
$$

Thus T-duality essentially exchanges the rank and the first Chern class of the D-brane.

\section{Abelian duality in 3d}

\subsection{The duality wall in $3 \mathrm{~d}$}

Consider a U(1) gauge theory on a Riemannian 3-manifold $M$ with a Euclidean action

$$
S=\frac{1}{2 e^{2}} \int_{M} F \wedge * F
$$

This theory is equivalent to a bosonic sigma-model on $M$ with target $S^{1}$. The basic field of this sigma-model is a scalar $\sigma$ with the identification $\sigma \sim \sigma+2 \pi$ and an action

$$
\frac{e^{2}}{8 \pi^{2}} \int_{M} d \sigma \wedge * d \sigma .
$$


The corresponding duality wall is given by an insertion of an operator

$$
\exp \left(\frac{-i}{2 \pi} \int_{W} A d \sigma\right)
$$

where $W$ is an oriented surface in $M$ splitting it into $M_{+}$and $M_{-}$. By convention, the orientation of $W$ agrees with that of $M_{-}$and disagrees with that of $M_{+}$. The fields $A$ and $\sigma$ are defined on $M_{-}$and $M_{+}$, respectively. Their boundary values on $W$ are unconstrained. Varying the action and requiring the vanishing of boundary terms in the variation gives the following matching conditions on $W$ :

$$
\begin{aligned}
\left.* F\right|_{W} & =\left.\frac{i e^{2}}{2 \pi} d \sigma\right|_{W}, \\
\left.F\right|_{W} & =\left.\frac{i e^{2}}{2 \pi} * d \sigma\right|_{W} .
\end{aligned}
$$

One can check that these conditions ensure the continuity of the stress-energy tensor across $W$. This shows that the wall operator is indeed topological.

The definition of the duality wall given above is somewhat imprecise, because the connection 1-form $A$ is defined only up to a gauge transformation. Formal integration by parts does not ameliorate the situation, because the expression

$$
\int_{W} F \sigma
$$

where $\sigma$ is defined up to a multiple of $2 \pi$, is also ill-defined. In fact, integration by parts makes the matters worse, because it creates an illusion that the wall operator depends only on $F$, not $A$, which is incorrect.

The most natural interpretation of the expression (4.1) is in terms of a connection on a $\mathrm{U}(1)$ gerbe over $W$. In the string theory context, a gerbe connection is also known as a B-field. For a brief review of the topology of the B-field see e.g. [9]. For our purposes, a gerbe connection is given by a collection of 2 -forms $B_{i}$ defined on charts $U_{i}$ of an open cover of $W$, where $i$ runs over the index set of the cover. The 2-forms satisfy gluing conditions on the overlaps $U_{i j}=U_{i} \bigcap U_{j}$ :

$$
B_{i}-B_{j}=d A_{i j}
$$

Here $A_{i j}$ are U(1) connection 1-forms on open sets $U_{i j}$. They obviously satisfy $A_{i j}=-A_{j i}$. As part of the definition of the gerbe connection, $A_{i j}$ are required to satisfy a gluing condition of their own on triple overlaps $U_{i j k}=U_{i} \bigcap U_{j} \cap U_{k}$ :

$$
A_{i j}+A_{j k}+A_{k i}=d f_{i j k}
$$

where $f_{i j k}$ are $\mathrm{U}(1)$-valued functions on triple overlaps completely antisymmetric with respect to indices $i j k$. Here we think about $\mathrm{U}(1)$ as $\mathbb{R} / 2 \pi \mathbb{Z}$ rather than as the unit circle in the complex plane, to avoid the annoying factors $\sqrt{-1}$ in the formulas. The functions $f_{i j k}$ must satisfy a cocycle condition on quadruple overlaps $U_{i j k l}$ :

$$
f_{i j k}+f_{j k l}+f_{k l i}+f_{l i j}=0 \bmod 2 \pi \mathbb{Z} .
$$


The gerbe itself is defined by functions $f_{i j k}$; the rest of the data defines a connection on this gerbe. It should be clear from the above description that a gerbe is a "higher analogue" of a line bundle.

Just like an ordinary connection on a line bundle assigns an element of $U(1)$ (the holonomy) to any loop in the manifold, a gerbe connection assigns an element of U(1) to any closed oriented two-dimensional submanifold. This number is usually called gerbe holonomy. $^{2}$ In our case, since $W$ is two-dimensional, the only submanifold of interest is $W$ itself.

The construction of gerbe holonomy is described in detail in [5]. In brief, one triangulates $W$ and considers an open cover whose charts are so-called open stars of the triangulation labeled by vertices of the triangulation. (An open star associated to a vertex is the interior of the union of all simplices containing this vertex.) Then one considers a cell decomposition dual to the triangulation. The 2-cells are labeled by vertices of the triangulation, 1-cells are labeled by edges of the triangulation, and 0-cells are labeled by simplices of the triangulation (they are baricenters of the simplices). Note that each 2-cell lies entirely within a single open chart and therefore can be naturally associated with this open chart. Similarly, each 1-cell belongs to the closure of precisely two 2-cells and belongs to the overlap of the corresponding open charts; therefore it can be naturally associated to an overlap of two charts. Finally, each 0 -cell lies in a unique triple overlap. To put it differently, each 2-form $B_{i}$ naturally lives on a particular 2-cell, each 1-form $A_{i j}$ naturally lives on a particular 1-cell, and each $f_{i j k}$ naturally lives on a particular 0-cell.

Now the gerbe holonomy is defined as follows. One integrates all $B_{i}$ over the corresponding 2-cells, all $A_{i j}$ over the corresponding 1-cells, evaluates $f_{i j k}$ on the corresponding 0 -cells and adds up the results. This gives an element of $\mathbb{R} / 2 \pi \mathbb{Z}$ which one can then multiply by $\sqrt{-1}$ and exponentiate. The resulting phase is the gerbe holonomy. One can show that the gerbe holonomy is independent of the choice of the triangulation.

Now we show that the expression $\frac{1}{2 \pi} A d \sigma$ can be naturally interpreted as a connection on a gerbe. We pick an open cover of $W$

$$
\left\{U_{i}, i \in \mathrm{S}\right\}
$$

such that all $U_{i}$ and all multiple overlaps are contractible. Recall that the gauge field $A$ is defined by a collection of 1 -forms $A_{i}$ on $U_{i}$ such that on double overlaps we have

$$
A_{i}-A_{j}=d g_{i j}
$$

where $g_{i j}$ are $\mathbb{R}$-valued transition functions. (The usual complex-valued transition functions are obtained by multiplying $g_{i j}$ by $\sqrt{-1}$ and exponentiating.) On triple overlaps they must satisfy

$$
g_{i j}+g_{j k}+g_{k i}=2 \pi m_{i j k}, \quad m_{i j k} \in \mathbb{Z} .
$$

The integers $m_{i j k}$ form a Cech 2-cocycle representing the first Chern class of the U(1) gauge bundle. Similarly, one can specify $\sigma$ by $\mathbb{R}$-valued functions $\sigma_{i}$ on $U_{i}$ which satisfy on double overlaps

$$
\sigma_{i}-\sigma_{j}=2 \pi r_{i j}, \quad r_{i j} \in \mathbb{Z} .
$$

\footnotetext{
${ }^{2}$ In string theory context, gerbe holonomy is the factor in the path-integral arising from the B-field.
} 
The integers $r_{i j}$ form a Cech 1-cocycle representing the cohomology class of the 1-form $d \sigma$.

To define a gerbe connection on $W$ we let

$$
B_{i}=\frac{1}{2}\left(A_{i} \frac{d \sigma_{i}}{2 \pi}+d A_{i} \frac{\sigma_{i}}{2 \pi}\right) .
$$

Then on double overlaps $U_{i j}$ we have

$$
B_{i}-B_{j}=\frac{1}{2}\left(d g_{i j} \frac{d \sigma_{j}}{2 \pi}+d A_{i} r_{i j}\right) .
$$

The right-hand side can be written as a total derivative

$$
\frac{1}{2} d\left(A_{i} r_{i j}+\frac{\sigma_{j}}{2 \pi} d g_{i j}+2 g_{i j} \frac{d \sigma_{j}}{2 \pi}\right) .
$$

We therefore let

$$
A_{i j}=\frac{1}{2}\left(A_{i} r_{i j}+\frac{\sigma_{j}}{2 \pi} d g_{i j}\right)+g_{i j} \frac{d \sigma_{j}}{2 \pi} .
$$

One can check that the 1 -forms $A_{i j}$ satisfy $A_{i j}=-A_{j i}$. One can also check that on triple overlaps we have

$$
A_{i j}+A_{j k}+A_{k i}=m_{i j k} d \sigma_{j}
$$

Hence we let

$$
f_{i j k}=m_{i j k} \sigma_{j} .
$$

This function is well-defined as a map from $U_{i j k}$ to $\mathbb{R} / 2 \pi \mathbb{Z}$, is completely antisymmetric with respect to all indices, and obviously satisfies the cocycle condition (4.4) on quadruple overlaps. Hence we have successfully defined a gerbe on $W$ and a connection on it. The duality wall will be defined as the holonomy of this gerbe connection.

One can modify the U(1) gauge theory by adding a Chern-Simons term to the action. This modification destroys the duality between the gauge theory and the sigma-model. In the duality wall formalism this comes about from the fact that the Chern-Simons term is not gauge invariant in the presence of a free boundary on which $F=d A$ is allowed to be nonzero. Thus the duality wall operator as defined above is not even gauge-invariant. One could try to rectify this by placing an extra degree of freedom on the wall which couples to the gauge field in an anomalous manner. For example, one could put a $2 \mathrm{~d}$ chiral gauge boson on $W$ which couples to $A$. But the resulting wall operator is not topological and does not define a duality transformation.

\subsection{Duality action on operators}

Let us give some examples demonstrating how the duality wall acts on operators in the dual theories. First of all, the matching conditions (4.2), (4.3) imply that the local operator $F$ is mapped to

$$
\frac{i e^{2}}{2 \pi} * d \sigma
$$

This is usually taken as the definition of the duality transformation. 
Another local operator is the 't Hooft operator. In $3 \mathrm{~d}$ it is a local operator (i. e. it is localized at a point $p \in M)$. It is a disorder operator defined by the condition that at the insertion point $p F$ is singular and satisfies

$$
\int_{W} \frac{F}{2 \pi}=m
$$

Here $W$ is a small 2-sphere centered at $p$. The integer $m$ is called the magnetic charge. The dual of the 't Hooft operator is the operator $\exp (-i m \sigma(p))$. To see this, we place the duality wall at $W$. Since $W$ is simply-connected, the map $\sigma$ can be thought of as an ordinary $\mathbb{R}$-valued function and the gerbe holonomy simplifies to

$$
\exp \left(\frac{-i}{2 \pi} \int_{W} F \sigma\right)=\exp (-i m \sigma) \text {. }
$$

Now the integral over $A$ is decoupled from the integral over $\sigma$, and therefore integration over $A$ produces an irrelevant constant.

Next we consider the action of duality on line operators in the gauge theory. The simplest such operator is the Wilson line operator

$$
W_{n}(\gamma)=\exp \left(i n \int_{\gamma} A\right)
$$

where $\gamma$ is a circle embedded into $M$, and $n \in \mathbb{Z}$ is the electric charge. The argument allowing to calculate its dual is essentially identical to the four-dimensional case discussed in section 2.3.1, only simplified because of the absence of a $\theta$-term in the action. First we are going to regularize the Wilson operator. Let $\mathcal{Z}_{\epsilon}$ be a tubular neighborhood of $\gamma$ of "thickness" $\epsilon$. Let $\psi_{n}$ be a harmonic map $M \backslash \mathcal{Z}_{\epsilon} \rightarrow S^{1}$ satisfying

$$
\left.* d \psi_{n}\right|_{\partial \mathcal{Z}_{\epsilon}}=0 \text { and } \int_{L} d \psi_{n}=2 \pi n,
$$

where $L$ is a small circle which has linking number 1 with $\gamma$. At this stage we assume that $\gamma$ is homologically trivial, so that the linking number is well-defined. (As in the $4 \mathrm{~d}$ case, if $\gamma$ is homologically nontrivial and there are no other Wilson loops inserted, the path-integral vanishes identically because the integrand is not invariant with respect to adding to the connection a flat connection with a nontrivial holonomy along $\gamma$.) The regularized Wilson loop is defined to be

$$
W_{n}^{\epsilon}(\gamma)=\exp \left(\frac{i}{2 \pi} \int_{\partial \mathcal{Z}_{\epsilon}} A \wedge d \psi_{n}\right)
$$

More generally, if several Wilson loops $\gamma_{1}, \ldots, \gamma_{K}$ with charges $n_{1}, \ldots, n_{K}$ are present simultaneously, the path-integral can be nonvanishing only if the following condition is satisfied:

$$
\sum_{i=1}^{K} n_{i}\left[\gamma_{i}\right]=0 .
$$

Here $\left[\gamma_{i}\right]$ denotes the homology class of $\gamma_{i}$. One can show then that on the complement of the tubular neighborhood $\mathcal{Z}_{\epsilon}$ of all the loops $\gamma_{i}$ there exists a harmonic map $\psi_{n}$ which satisfies

$$
\left.* d \psi_{n}\right|_{\partial \mathcal{Z}_{\epsilon}}=0, \quad \int_{L_{i}} d \psi_{n}=2 \pi n_{i},
$$


where $L_{i}$ is a small circle which winds once around $\gamma_{i}$.

The 1-form $d \psi_{n}$ can be seen as a magnetic field created by $K$ superconducting wires filling the tubular neighborhoods of $\gamma_{i}, i=1, \ldots, K$, such that the $i^{\text {th }}$ wire carries electric current $2 \pi n_{i}$. The multivalued function $\psi_{n}$ is the "magnetic potential."

Having regularized the Wilson operator by transforming it into an integral over $\partial \mathcal{Z}_{\epsilon}$, we place the duality wall on this surface and notice that the Wilson loop and the wall operator combine into a factor

$$
\exp \left(-\frac{i}{2 \pi} \int_{W} A\left(d \sigma-d \psi_{n}\right)\right)
$$

where $W=\partial \mathcal{Z}_{\epsilon}$ is the $S$-duality wall. ${ }^{3}$ Then, as in four-dimensional case, we shift the integration variables:

$$
\sigma=\sigma^{\prime}+\psi_{n}
$$

and note that in terms of $\sigma^{\prime}$ the wall operator (4.7) becomes

$$
\exp \left(-\frac{i}{2 \pi} \int_{W} A d \sigma^{\prime}\right)
$$

while the bulk action splits in two parts

$$
\frac{1}{2 e^{2}} \int_{M} d \sigma^{\prime} \wedge * d \sigma^{\prime}+\frac{1}{2 e^{2}} \int_{M} d \psi_{n} \wedge * d \psi_{n}
$$

As in the four-dimensional case, we recognize the situation as a $S$-duality wall separating two theories with no operator insertions, with weights of different topological sectors modified by an overall multiplicative factor. We eliminate the field $A$ by sending $\epsilon$ to zero; then we are left with a path-integral for $\sigma^{\prime}$ with the standard action and the multiplicative factor which we can reabsorb into the action. The new action is

$$
\frac{1}{2 e^{2}} \int_{M} d\left(\sigma^{\prime}+\psi_{n}\right) \wedge * d\left(\sigma^{\prime}+\psi_{n}\right) .
$$

Here $\sigma^{\prime}$ is nonsingular at $\gamma$, while $\psi_{n}$ has a singularity of the form

$$
n \phi+\text { regular terms. }
$$

Here we identified the neighborhood of $\gamma$ with $S^{1} \times D^{2}$, where $D^{2}$ is a small disc in $\mathbb{R}^{2}$ with a radial coordinate $r$ and angular coordinate $\phi$. Since the action depends only on the sum $\sigma=\sigma^{\prime}+\psi_{n}$, we may say that the dual of $W_{n}$ is the prescription to integrate over all fields $\sigma$ such that $\sigma$ has the same singularity at $\gamma$ as $\psi_{n}$. This defines a disorder operator in the theory of the field $\sigma$.

There is also a natural disorder line operator in the $3 \mathrm{~d}$ gauge theory. It is the reduction of the Gukov-Witten-type surface operator [8] from 4d to 3d (with the reduced dimension taken to be along the surface). It is defined by the condition

$$
F=\alpha \delta_{\gamma},
$$

\footnotetext{
${ }^{3}$ The gerbe holonomy again simplifies in this case because the $\mathrm{U}(1)$ bundle is trivial when restricted to $W$ and the 1-form $A$ can be thought of as an ordinary 1-form on $W$.
} 
where $\delta_{\gamma}$ is a delta-current supported on $\gamma$. Another way to define it is to say that the holonomy of $A$ along a small circle linking $\gamma$ approaches $\exp (2 \pi i \alpha)$ as the size of the circle shrinks to zero. The second definition makes it clear that $\alpha \in \mathbb{R}$ is defined modulo $\mathbb{Z}$.

To find its dual, we again place the duality wall at the boundary $W$ of the tubular neighborhood $\mathcal{Z}_{\epsilon}$ of $\gamma$. Up to regular terms, the restriction of $A$ to $W$ is given by $\alpha d \psi_{1}$, where $\psi_{1}: M \backslash \mathcal{Z}_{\epsilon} \rightarrow S^{1}$ has been defined above. Hence up to terms which vanish in the limit $\epsilon \rightarrow 0$ the duality wall operator becomes

$$
\exp \left(\frac{-i \alpha}{2 \pi} \int_{W} d \psi_{1} d \sigma\right)
$$

This is a regularization of

$$
\exp \left(i \alpha \int_{\gamma} d \sigma\right)
$$

One can describe this operator in words as follows. Consider a U(1) gauge field on $S^{1}$ whose holonomy is $\exp (i \alpha)$. Given a map $\sigma: M \rightarrow S^{1}$, we may pull back this $\mathrm{U}(1)$ gauge field to $M$ and evaluate its holonomy along $\gamma \subset M$. The resulting observable is precisely (4.8). One may call this observable a Wilson line operator; they have been introduced by Rozansky and Witten in the context of topological sigma-models in $3 \mathrm{~d}$ [11].

\subsection{Duality action on boundary conditions}

The two most natural boundary conditions in the 3d gauge theory are the Dirichlet and Neumann boundary conditions. The Dirichlet condition is analogous to the PEC condition in $4 \mathrm{~d}$ : we require $\left.A\right|_{\partial M}$ to be equal to a fixed flat connection on $\partial M$. Thus $F$ vanishes when restricted to the boundary. The Neumann boundary condition corresponds to keeping $\left.A\right|_{\partial M}$ unconstrained and imposing

$$
\left.* F\right|_{\partial M}=0
$$

On the quantum level the Neumann condition has a modulus: we can add to the action a boundary term

$$
\frac{i \theta}{2 \pi} \int_{\partial M} F
$$

Clearly, the parameter $\theta$ is defined modulo $2 \pi$.

The duality maps the Dirichlet condition in the gauge theory to the Neumann condition in the sigma-model:

$$
\left.* d \sigma\right|_{\partial M}=0 .
$$

This boundary condition has moduli: one can pick an arbitrary flat connection $a$ on $\partial M$ and add to the action a boundary term

$$
\frac{i}{2 \pi} \int_{\partial M} a d \sigma
$$

(More precisely, we should understand the corresponding phase factor in the path-integral as the holonomy of a gerbe.) Using the duality wall argument one can check that duality 
maps the flat connection $\left.A\right|_{\partial M}$ to $-a$. Indeed, as the duality wall merges with the Neumann boundary in the scalar field theory, the boundary action becomes

$$
\frac{i}{2 \pi} \int_{W}(A+a) d \sigma
$$

If we separate $\sigma$ into a topologically-trivial and topologically-nontrivial parts, integration over the topologically-trivial part gives a functional delta-function which forces $F=d A$ to vanish on the boundary, while integration over the topologically-nontrivial part forces the holonomy of the flat connection $A+a$ to vanish.

The duality also maps the Neumann condition in the gauge theory to the Dirichlet condition in the sigma-model:

$$
\left.d \sigma\right|_{\partial M}=0 .
$$

This means that $\sigma$ is a constant $\sigma_{0}$ on any connected component of $\partial M$. The modulus $\sigma_{0}$ is dual to the modulus $\theta$ in the gauge theory. Indeed, as the duality wall merges with the Dirichlet boundary in the scalar field theory, it becomes a boundary term in the action

$$
\frac{i}{2 \pi} \int_{\partial M} F \sigma_{0}
$$

We may also consider more general boundary conditions in the gauge theory involving boundary degrees of freedom coupled to the gauge field $A$ in a gauge-invariant manner. For example, we may consider a sigma-model with target $X$ which admits a U(1) isometry and promote this symmetry to a gauge symmetry by replacing ordinary derivatives with covariant derivatives. To determine the dual of this boundary condition, we place the duality wall infinitesimally close to the boundary. The gauge field $A$ in the gap between the wall and the boundary becomes effectively two-dimensional, and the boundary action becomes

$$
S_{b d r y}(\phi, A)+\frac{i}{2 \pi} \int_{\partial M} F \sigma
$$

Since the $2 \mathrm{~d}$ gauge field has no kinetic term, one can regard the boundary theory as the strong-coupling limit of the ordinary gauged sigma-model with target $X$, with the $2 \mathrm{~d}$ theta-angle promoted to a field $\left.\sigma\right|_{\partial M}$.

\section{References}

[1] M.V. Berry, Quantal phase factors accompanying adiabatic changes, Proc. Roy. Soc. Lond. A 392 (1984) 45 [SPIRES].

[2] J. Fuchs, C. Schweigert and K. Waldorf, Bi-branes: Target space geometry for world sheet topological defects, J. Geom. Phys. 58 (2008) 576 [hep-th/0703145] [SPIRES].

[3] J. Fuchs, M.R. Gaberdiel, I. Runkel and C. Schweigert, Topological defects for the free boson CFT, J. Phys. A 40 (2007) 11403 [arXiv:0705.3129] [SPIRES].

[4] D. Gaiotto and E. Witten, S-duality of Boundary Conditions In $N=4$ Super Yang-Mills Theory, arXiv:0807.3720 [SPIRES]. 
[5] K. Gawedzki, Topological actions in two-dimensional quantum field theories, in Nonperturbative quantum field theories, eds. G. 't Hooft, A. Jaffe, G. Mack, P.K. Mitter and R. Stora, NATO series 185, Plenum Press (1988) p. 101-141.

[6] P.B. Gilkey, Invariance theory, the heat equation and the Atiyah-Singer index theorem, Publish or Perish, Wilmington (1984).

[7] P.B. Gilkey, J.V. Leahy and J. Park, Spinors, spectral geometry and Riemannian submersions, Lecture Notes Series 40, Research Institute of Mathematics, Global Analysis Research Center, Seoul National University (1998), available at the EMIS server http://www.emis.de/monographs/GLP/index.html.

[8] S. Gukov and E. Witten, Gauge theory, ramification and the geometric Langlands program, hep-th/0612073 [SPIRES].

[9] A. Kapustin, D-branes in a topologically nontrivial B-field, Adv. Theor. Math. Phys. 4 (2000) 127 [hep-th/9909089] [SPIRES].

[10] A. Kapustin and E. Witten, Electric-magnetic duality and the geometric Langlands program, hep-th/0604151 [SPIRES].

[11] L. Rozansky and E. Witten, Hyper-Kähler geometry and invariants of three-manifolds, Selecta Math. 3 (1997) 401 [hep-th/9612216] [SPIRES].

[12] G. Sarkissian and C. Schweigert, Some remarks on defects and T-duality, Nucl. Phys. B 819 (2009) 478 [arXiv:0810.3159] [SPIRES].

[13] B. Simon, Holonomy, the quantum adiabatic theorem and Berry's phase, Phys. Rev. Lett. 51 (1983) 2167 [SPIRES].

[14] E. Witten, SL(2,Z) action on three-dimensional conformal field theories with Abelian symmetry, hep-th/0307041 [SPIRES].

[15] R. Zucchini, Four dimensional Abelian duality and $\mathrm{SL}(2, \mathbb{Z})$ action in three dimensional conformal field theory, Adv. Theor. Math. Phys. 8 (2005) 895 [hep-th/0311143] [SPIRES]. 